\title{
Bow wave from ultra-intense electromagnetic pulses in plasmas
}

\author{
T. Zh. Esirkepov, ${ }^{1}$ Y. Kato, ${ }^{2}$ and S. V. Bulanov ${ }^{1, \text { * }}$ \\ ${ }^{1}$ Kansai Photon Science Institute, JAEA, Umemidai 8-1, Kizugawa, Kyoto 619-0215, Japan \\ ${ }^{2}$ The Graduate School for the Creation of New Phototnics Industries, \\ Kurematsucho 1955-1, Nishiku, Hamamatsu, Shizuoka 431-1202, Japan
}

\begin{abstract}
We show a new effect of the bow wave excitation by an intense short laser pulse propagating in underdense plasma. Due to spreading of the laser pulse energy in transverse direction, the bow wave causes a large-scale transverse modulation of the electron density. This can significantly increase the electric potential of the wake wave since the wake wave is generated in the region much wider than the laser pulse waist.
\end{abstract}

PACS numbers: 52.38.-r, 52.38.Kd, 52.65.Rr

Bow waves are observed at various conditions in fluids, gases and plasmas. A bow wave is formed in the water at the bow of a moving ship [1 in addition to Kelvin waves left behind; it defines the outer boundary of the ship wakes. Reducing this effect is an important aim of shipbuilding, since bow waves take away a portion of the ship energy and can damage shore facilities. In air, a spectacular bow wave accompanied with a vapour cone manifesting the Prandtl-Glauert singularity [2] is excited by an aircraft when it breaks the sound barrier. In space, plasma bow waves appear at various scales. A bow shock is formed where the Earth's magnetosphere is squeezed by the solar wind flow 3]. The largest ever seen bow waves were observed in the collision of galaxies by the Spitzer Space Telescope [4. As these examples show, bow waves lead to a vast range of physical processes such as a transverse transfer of momentum and matter and particle acceleration.

As is well known, an intense short laser pulse propagating in underdense plasma excites wake waves, similarly to a moving ship producing the Kelvin waves. This analogy underlying a fruitful exchange of ideas between hydrodynamics and plasma physics can be extended further. In this Letter we demonstrate that a laser pulse can excite a bow wave in collisionless plasma. Like a bow wave from a ship, the bow wave from a laser pulse spread aside the energy and the momentum of the laser pulse. Although generation of shock waves in laser plasmas has been known shortly after the first laser was built [5, to our best knowledge, the bow waves formed by relativistic electrons at the ultra-short laser pulse propagating in plasma have not been discussed so far.

The laser-driven wake waves in plasmas are used in the laser wake field accelerator (LWFA) [6] and the flying mirror (FM) [7] concepts. A tightly focused and sufficiently intense laser pulse excites a wake field [6] in the so-called blowout regime [8, 9] or bubble regime [10, where, in addition to a longitudinal push, the laser pulse expells electrons also in transverse direction, forming a cavity void of electrons in the first period of the wake wave. Thus a finite waist of the laser pulse results in a transverse motion of electrons, which leads to a transverse wave-breaking [11] underlying one of the mechanisms of self-injection of electrons into the accelerating phase of the wake wave [8]. We show that when the waist of a sufficiently intense laser pulse is less than the wake wave length, the expelled electrons can not be confined near the cavity and their transverse outflow forms a bow wave. The bow wave should be distinctly seen in the limit of high intensity and narrow waist laser pulses and low-density plasma, which correspond to the conditions for generating higher energy electrons by laser wakefield acceleraion [8, 12.

*Also at A. M. Prokhorov Institute of General Physics of RAS, Moscow, Russia. 


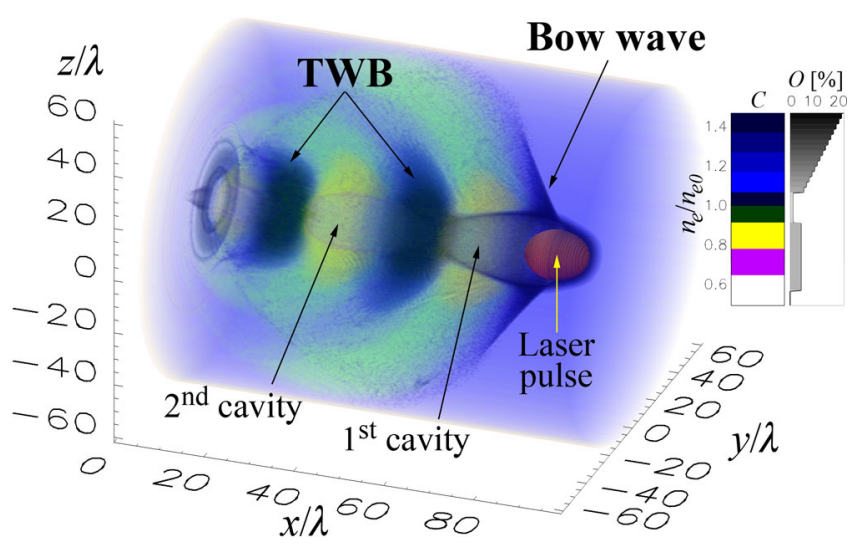

FIG. 1: (color). Electron density (coded into color, $C$, and opacity, $O$, with ray-tracing) in the wake of the laser pulse, which is represented by the electromagnetic energy density isosurface $\left(E^{2}+B^{2}\right)\left(m_{e} \omega c / e\right)^{2}=4($ red $)$, at $t=100 \times 2 \pi / \omega$.

We present the results of three-dimensional (3D) particle-in-cell (PIC) simulations with the Relativistic ElectroMagnetic Particle-mesh (REMP) code [15] performed on Altix 3700 supercomputer at JAEA-Tokai. The laser pulse with the initial intensity $I=6 \times 10^{19} \mathrm{~W} / \mathrm{cm}^{2} \times(1 \mu \mathrm{m} / \lambda)^{2}$, corresponding to the dimensionless amplitude $a=e E_{0} / \omega m_{e} c=6.62$, propagates along the $x$-axis; it is linearly polarized in the direction of the $y$-axis and has the size of $\ell_{x} \times \ell_{y} \times \ell_{z}=10 \lambda \times 10 \lambda \times 10 \lambda$. The pulse is incident on a fully ionized plasma slab with the electron density $n_{e}=1.14 \times 10^{18} \mathrm{~cm}^{-3} \times(1 \mu \mathrm{m} / \lambda)^{2}$. Here $\lambda$ and $\omega$ are the laser wavelength and frequency, $e$ and $m_{e}$ are the charge and mass of electron, $E_{0}$ is the magnitude of the laser pulse electric field, and $c$ is the speed of light in vacuum. We consider the electron bow wave, when the ion response can be neglected, i. e. the ion-to-electron mass ratio is $m_{i} / m_{e} \rightarrow \infty$. The simulation grid dimensions are $4000 \times 992 \times 992$ along $x, y$ and $z$ axes; the grid mesh sizes are $d x=\lambda / 32, d y=d z=\lambda / 8$; total number of quasi-particles is $2.3 \times 10^{10}$. For observing the bow wave, the number of quasi-particles must be sufficiently high since the density of quasi-particles expelled in transverse direction drops with a distance $r$ from the axis as $r^{-2}$ (in 3D configuration). In the figures, the time and spatial units are the laser wave period and wavelength, respectively.

The laser pulse excites a strong wake wave, whose first period forms a cavity completely void of electrons, Figs. 1 . 2. In addition to a longitudinal push, the laser pulse drives electrons also in transverse direction, forming the bow wave. As seen in Fig. 2(a), the longitudinal electric field $\left(E_{x}\right)$ is formed in a region which is much greater in transverse direction than the cavity width corresponding to the first period of the wake wave. Due to this, the electric potential, $\varphi$, is not limited by the transverse size of the cavity. We note that at present conditions the length of the cavity (i. e., its size along $x$-axis) is 1.5 times greater than its width (the size in transverse direction). In the regimes not favourable for the bow wave formation, e. g. when the bow wave does not detach from the cavity, the electric potential in the cavity is determined by the smallest dimension of the cavity, either longitudinal or transverse.

A strong transverse wave-breaking (TWB) 11 forms the transverse outflow of electrons, which increases the electric potential in the subsequent periods of the wake wave, similarly to the bow wave. However, in contrast to the bow wave, which arises because electrons are directly pushed by ponderomotive force of the laser pulse, the TWB wave is due to an electrostatic field of the wake wave itself caused by the finite waist of the laser pulse.

The electron motion in the wake wave, bow wave as well as motion due to TWB, forms a transient electron density modulations with associated electromagnetic field. Fig. 3 shows the magnetic field co-moving with the cavities. The polarity of the magnetic field corresponds to the forward motion of a negatively charged object. The strong magnetic field associated with the region of the electron density compression due to TWB can be described in the approximation of a Lienard-Wiechert potential of a moving point charge [16.

As shown in the electron phase plot, Fig. 4(c), the transverse momentum of the electrons undergone TWB behind the first cavity is higher than that of electrons in the bow wave. This is due to Coulomb explosion of the electron flow concentrated near the axis, Fig. $4(a, b)$.

The shape of the cavity in the electron density behind the laser pulse can be deduced from the fact that, in the limit of a well-developed bow wave, all the electrons on a way of the laser pulse are pushed aside forming a multiflow motion owing to the collisionless nature of the low density plasma. The region void of electrons is positively charged and attracts the unpertubed electrons from aside. The transverse (radial) component of the electric field near the axis is proportional to the radius, $E_{\perp}=2 \pi n_{e} e r$. It is easy to show that for the laser pulse waist smaller than $2 \pi c a / \omega_{p e}$, where $\left.\omega_{p e}=4 \pi n_{e} e^{2} / m_{e}\right)^{1 / 2}$ is the Langmuir frequency, the bulk electrons remain nonrelativistic. The electrons start to perform an oscillation with frequency $\Omega \propto \omega_{\text {pe }}$, determined by the geometry of the cavity. After one quarter of the oscillation period, $\pi / 2 \Omega$, they reach the cavity axis. Therefore the characteristis shape of the cavity formed in the 


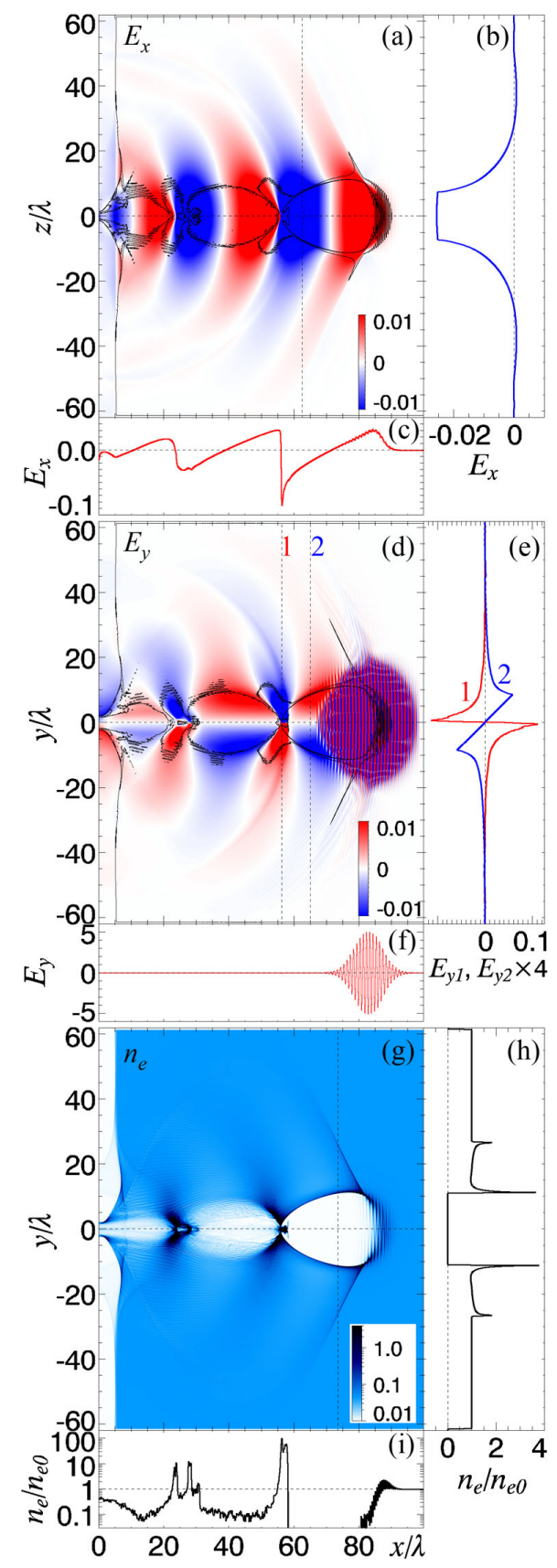

FIG. 2: (color). 2D cross-sections of the electric field components $E_{x}$ (a) and $E_{y}$ (d) and of the electron density (g) at $t=100 \times 2 \pi / \omega$. Vertical (b,e,h) and horizontal (c,f,i) $1 \mathrm{D}$ cross sections of the E-field and density correspond to the positions shown by the dashed lines. In $(\mathrm{a}, \mathrm{d})$ the electron density curves are drawn by the black curves for $n_{e} / n_{e 0}=0.75,1.5$.

first wake wave period can be approximated by the curve

$$
r=R_{c} \cos \left(\Omega\left(t-x / v_{p h}\right)\right)
$$

for $-\pi / 2<\Omega\left(t-x / v_{p h}\right)<0$, where $v_{p h}$ is the phase velocity of the wake wave equal to the group velocity, $v_{g}$, of the laser pulse.

Comparing the kinetic energy of the expelled electrons, $\approx a m_{e} c^{2}$, with their potential energy at the cavity periphery, $e \varphi=\pi n_{e} e^{2} R_{c}^{2}$, where $R_{c}$ is the cavity radius, we find a condition of the bow wave formation: $R_{c}<2 c a^{1 / 2} / \omega_{p e}=$ $(\lambda / \pi)\left(a n_{c r} / n_{e}\right)^{1 / 2}$. If we assume that the cavity transverse size is not greater than the laser pulse waist, $D_{\text {las }} \geq R_{c}$, then the condition of the bow wave formation can be rewritten in a stronger form as $D_{\text {las }} \leq 4 c a^{1 / 2} / \omega_{p e}$. In the 


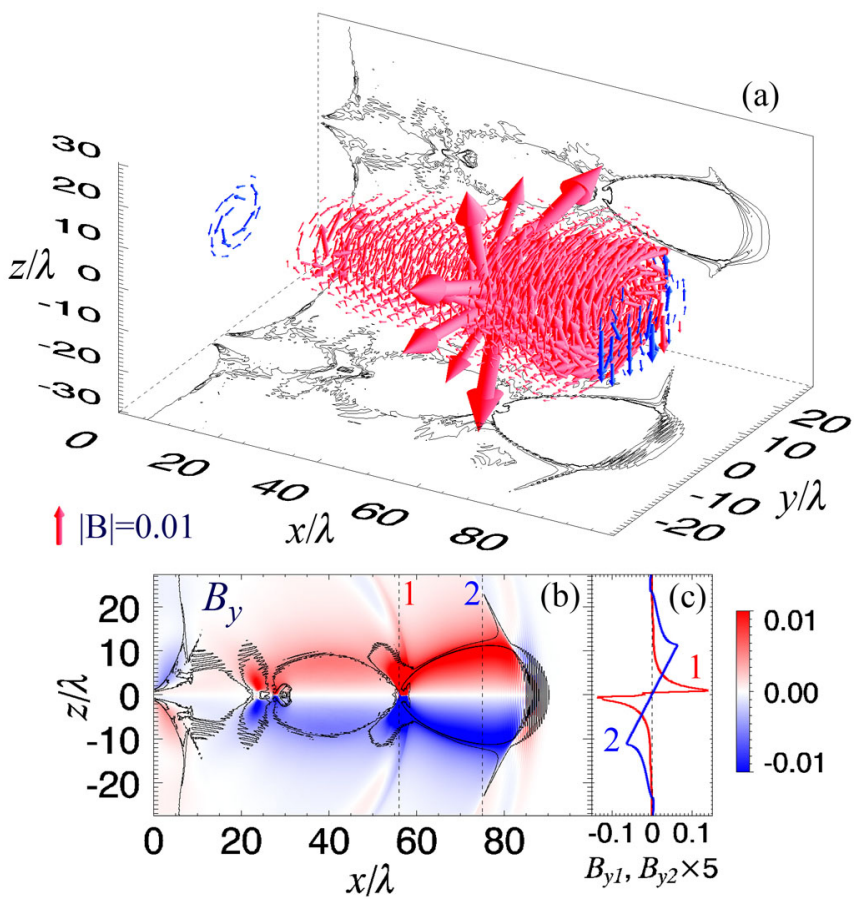

FIG. 3: (color). (a) Magnetic field, B, around the cavities; laser frequency is filtered out. Red arrows for clockwise, blue - for counterclockwise vector $B$. On side panels $-2 \mathrm{D}$ cross-sections of the electron density curves for $n_{e} / n_{e 0}=0.8,1.4,2,2.6$. (b,c) $2 \mathrm{D}$ and $1 \mathrm{D}$ cross section of $B_{y}$ with electron density curves as in Fig. 2 Both frames for $t=100 \times 2 \pi / \omega$.

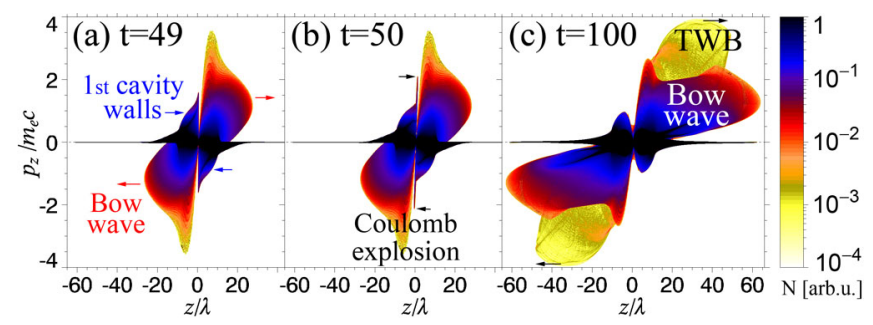

FIG. 4: (color). Electron phase space projection onto $\left(z, p_{z}\right)$ plane.

opposite limit, $D_{\text {las }} \gg 4 c a^{1 / 2} / \omega_{\text {pe }}$, the momentum acquired by electrons from the laser pulse is not enough for escaping in transverse direction, so the bow wave is closely attached to the cavity boundary.

As is well known, in one-dimensional (1D) case the laser pulse energy depletion length is given by $l_{\text {dep }} \sim l_{l a s}\left(\omega / \omega_{p e}\right)^{2}$, where $l_{\text {las }}$ is the laser pulse length, which comes from the balance of the laser energy and the energy left in the wake wave behind the pulse, when it is assumed that the electron energy is of the order of $a^{2} m_{e} c^{2} / 2$ 17. In the $3 \mathrm{D}$ configuration, when the laser pulse is tightly focused, $D_{\text {las }} \leq 4 c a^{1 / 2} / \omega_{p e}$, so that the bow wave is generated, the kinetic energy of electrons being expelled in transverse direction is $\approx a m_{e} c^{2}$, which results in the estimation for the depletion length: $l_{\text {dep }} \sim a l_{\text {las }}\left(\omega / \omega_{p e}\right)^{2}$, i. e. by the factor $a$ larger than in the $1 \mathrm{D}$ case.

In the LWFA concept [6] the energy of the electron accelerated by the wake field scales as

$$
\mathcal{E} \approx 2 e \Delta \varphi \gamma_{p h}^{2}
$$

where $\Delta \varphi=\varphi_{\max }-\varphi_{\text {min }}$ is the potential difference in the wake field and $\gamma_{p h}=\left(1-v_{p h}^{2} / c^{2}\right)^{-1 / 2}$ is the Lorentz factor corresponding to the wake wave phase velocity. In the $1 \mathrm{D}$ configuration the electromagnetic wave propagates with the group velocity, $v_{g}$, corresponding to $\gamma_{g}=\left(1-v_{g}^{2} / c^{2}\right)^{-1 / 2}=\gamma_{p h} \simeq a^{1 / 2} \omega / \omega_{p e}$ in the limit $a \gg 1$. For the optimal conditions of the acceleration, $e \Delta \varphi / m_{e} c^{2} \simeq a^{2} / 2$, thus we obtain 13 ]

$$
\mathcal{E}^{(1 D)} \approx a^{3} \frac{\omega^{2}}{\omega_{p e}^{2}} m_{e} c^{2} .
$$


Multi-dimensional effects modify the scaling law of the electron acceleration. In the cavity, formed behind a tighly focused laser pulse, close to the threshold of the bow wave formation, the electrostatic potential is mainly determined by the cavity transverse size, $\Delta \varphi \approx \varphi \simeq \pi n_{e} e D_{\text {las }}^{2} / 4$. For a narrow laser pulse its group velocity becomes strongly dependent on its waist, $\gamma_{p h} \simeq D_{\text {las }} / \lambda$. The laser pulse waist, in its turn, depends on the pulse evolution, i. e. selffocusing and electron density cavity formation. We find that the energy of electrons accelerated in the wake wave generated by a sufficiently strong, $a \gtrsim e \varphi / m_{e} c^{2}=(\pi / 2)^{2}\left(D_{\text {las }} / \lambda\right)^{2}\left(\omega_{\text {pe }} / \omega\right)^{2}$, and narrow, $D_{\text {las }} \lesssim 4 c a^{1 / 2} / \omega_{\text {pe }}$, laser pulse, does not depend on the laser pulse amplitude [18]

$$
\mathcal{E}^{(3 D)} \approx \frac{\pi^{2}}{2} \frac{D_{l a s}^{4}}{\lambda^{4}} \frac{\omega_{p e}^{2}}{\omega^{2}} m_{e} c^{2}
$$

We note that here the condition for $a$ implies $\mathcal{E}^{(3 D)} \lesssim 8 \pi^{-2} a^{2}\left(\omega / \omega_{p e}\right)^{2} m_{e} c^{2}$.

The shape of an outer boundary of the bow wave can be determined in the limit of a small density of electrons forming the bow wave, when these electrons can be treated as test particles. In this approximation, particles acquire initial momentum near the axis and then move in a potential of a positively charged semi-infinite wire of the cavity radius, $R_{c}$, propagating with velocity $v_{p h}$. This motion is described by the Hamiltonian $H=\left(m_{e}^{2} c^{4}+p_{x}^{2} c^{2}+p_{y}^{2} c^{2}\right)^{1 / 2}-$ $v_{p h} p_{x}+e \sigma \ln \left[\xi+\left(\xi^{2}+y^{2}\right)^{1 / 2}\right]$, for $y \geq R_{c}$. On the particle trajectory in phase space the Hamiltonian is equal to $H_{0}=\left(m_{e}^{2} c^{4}+p_{x 0}^{2} c^{2}+p_{y 0}^{2} c^{2}\right)^{1 / 2}-v_{p h} p_{x 0}+e \sigma \ln \left[y_{0}\right]$, where $\xi=x-v_{p h} t, \sigma \approx \pi n_{e} e R_{c}^{2}$ is the linear density of the wire, $p_{x 0}$ and $p_{y 0}$ are the initial momentum components at $y_{0}=R_{c}$. Neglecting the longitudinal momentum and assuming $p_{y 0}=a m_{e} c$, we obtain $y_{\max } \simeq R_{c} \exp \left[\left(1+a^{2}\right)^{1 / 2}\left(2 c / R_{c} \omega_{p e}\right)^{2}\right]$. Near the axis the outer boundary of the bow wave can be approximated by the straight line $v_{\perp 0}\left(x-v_{g} t\right)+\left(v_{g}-v_{\| 0}\right) y=0$, where $v_{\| 0}$ and $v_{\perp 0}$ are the components of the initial velocity of expelled electrons. The inclination of the line is determined by the expression $\tan \theta=d y / d x=v_{\perp 0} /\left(v_{g}-v_{\| 0}\right)$. Extrapolating the approximation of the outer boundary of the bow wave by a line over the distance equal to the half-length of the cavity, $\ell_{c} / 2$, we can estimate an addition to the cavity potential due to the bow-wave: $\varphi^{(B w)} \approx \sigma\left(1+\ln \left[\ell_{c} \tan \theta / 2 R_{c}\right]\right)$.

Among various effects associated with the bow wave we notice the following. In the multi-species underdense plasma, on the ion time scale the bow wave structure becomes enriched by contribution from the ion dynamics. The bow wave has a complex structure, where modulations of lighter components develop faster, determining the collisionless shock wave structure [5, 19]. The ion component of the bow wave is accelerated due to the laser ponderomotive potential and due to moving potential of the electron component of the bow wave. Transverse electric currents, associated with the bow wave from a laser pulse with strongly squeezed focal spot, should form large-scale quasi-static magnetic field and, due to instabilities, eventually should take a shape of separated jets. A portion of the transition radiation emitted at the plasma boundary interface [20] can be attributed to a change of the electric charge density, associated with the bow wave propagation, especially in a non-uniform plasma.

In conclusion, a tightly focused intense laser pulse propagating in underdense plasma, in addition to wake wave generation, excites bow waves and TWB waves, which spread the laser pulse energy in transverse direction. The effects of the bow and TWB waves excitation are essentially multidimensional. The bow wave increases the electric potential of the wake wave in comparison with the regimes where the bow wave can not detach from the cavity. The bow wave facilitates the transverse wave-breaking [11] which causes the self-injection of electrons into the accelerating phase of the wake field. In its turn, the wave-breaking forms a transverse flow of electrons similar to the electron motion in the bow wave.

Recent advances in LWFA 8, 12 reveal a tendency to use greater laser intensity and lower plasma density, aiming at higher energy of fast electrons. This makes the bow wave excitation inevitable. In such the regimes the multidimensional effects should modify the scaling laws of LWFA acceleration based on a one-dimensional approximation [6, 9, 13, or on the assumption that the cavity size is determined by the laser pulse amplitude [14.

\section{Acknowledgments}

This work is supported by the Grant-in-Aid for Scientific Research (A), 20244065, 2008 from MEXT (Japan) and VITP-ELI (France).

[1] H. Lamb, Hydrodynamics (Cambridge University Press, 1993, ISBN 0521458684).

[2] B. Lautrup, Physics of Continuous Matter: Exotic and Everyday Phenomena in the Macroscopic World (IOP Publishing, 2005, ISBN 0750307528). 
[3] N. Meyer-Vernet, Basics of the Solar Wind (Cambridge University Press, 2007, ISBN 0521814200).

[4] P. N. Appleton, et al., Astrophys. J. Lett. 639, L51 (2006).

[5] D. W. Koopman and D. A. Tidman, Phys. Rev. Lett. 18, 533 (1967).

[6] T. Tajima and J. M. Dawson, Phys. Rev. Lett. 43, 267 (1979).

[7] S. V. Bulanov, et al., Phys. Rev. Lett. 91, 085001 (2003).

[8] S. P. D. Mangles, et al., Nature (London) 431, 535-538 (2004); C. G. R. Geddes, et al., ibid. 431, 538-541 (2004); J. Faure, et al., ibid. 431, 541-544 (2004).

[9] W. Lu, et al., Phys. Rev. Lett. 96, 165002 (2006).

[10] A. Pukhov and J. Meyer-Ter-Vehn, Appl. Phys. B 74, 355 (2002).

[11] S. V. Bulanov, et al., Phys. Rev. Lett. 78, 4205 (1997).

[12] W. P. Leemans, et al., Nature Phys. 2, 696-699 (2006); T. Kameshima, et al., Applied Physics Express 1, 066001 (2008); S. Karsch, et al., New J. Phys. 9, 415 (2007); N. A. M. Hafz, et al., Nature Photonics 2, 571 (2008).

[13] E. Esarey and P. Sprangle, IEEE Trans. on Plasma Science 24, 252 (1996).

[14] I. Kostyukov, et al., Phys. Plasmas 11, 5256 (2004); S. Gordienko and A. Pukhov, ibid. 12, 043109 (2005).

[15] T.Zh.Esirkepov, Comput. Phys. Comm. 135, 144 (2001).

[16] T. V. Liseikina, et al., Phys. Rev. E 60, 5991 (1999).

[17] S. V. Bulanov, et al., Phys. Fluids B 4, 1935 (1992).

[18] S. V. Bulanov, Plasma Phys. Control. Fusion 48, B29 (2006).

[19] R. Z. Sagdeev and C. F. Kennel, Sci. Am. 264, 106 (1991); L. Romagnani, et al., Phys. Rev. Lett. 101, 025004 (2008).

[20] W. P. Leemans, et al., Phys. Rev. Lett. 91, 074802 (2003); Z.-M. Sheng, et al., Phys. Rev. E 69, 025401 (2004). 